\title{
Dorfentwicklung in der Lommatzscher Pflege
}

\author{
Gerhard Doleschal
}

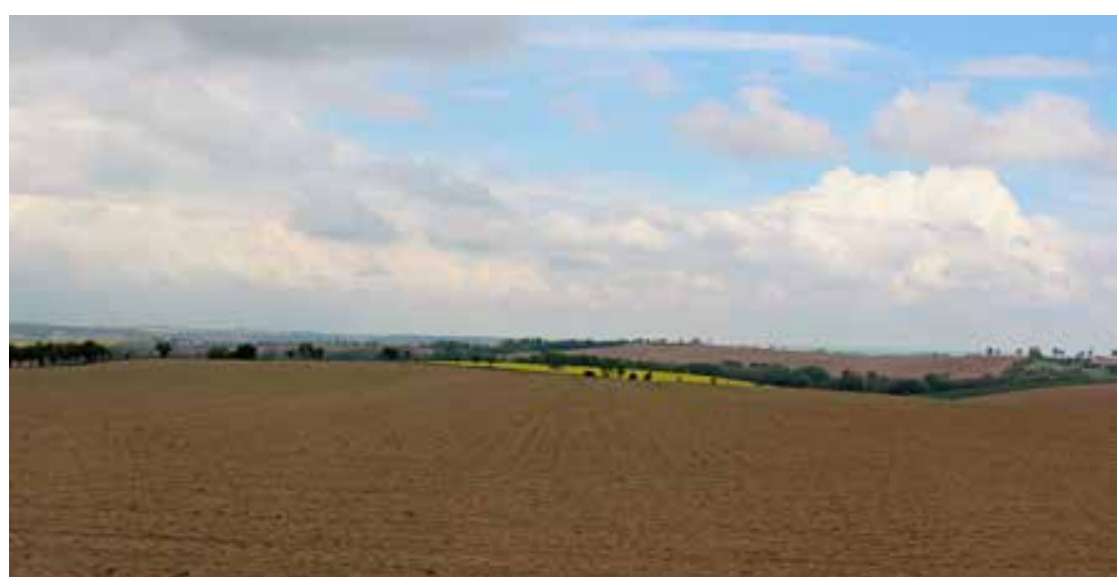

Die Lommatzscher Pflege wird von Kritikern als „ausgeräumte Agrarsteppe" beschrieben. Foto: Matthias Donath

Die Lommatzscher Pflege war, aufgrund des guten Bodens, geprägt von kleinen Dörfern und Drei- und Vierseithöfen mit einer Größe von 20 bis 70 Hektar, wobei Höfe über 50 Hektar die Ausnahme darstellten. Diese Höfe ernährten nicht nur die eigene Familie, sondern auch eine Reihe von Häuslern, die in den Gütern arbeiteten - direkt oder als Handwerker indirekt. Die Häuser der Häusler waren Fachwerkhäuser. Die Außenwände des Erdgeschosses errichtete man mit Feld- oder Bruchsteinen. Bei der Dachdeckung überwog in der Region der Dachziegel. Vereinzelt war eine Schieferdeckung anzutreffen.

Die Bewirtschaftung erfolgte in einem ausgewogenen Verhältnis von Tier- und Pflanzenwirtschaft. Da Düngemittel teuer waren, wurde größter Wert auf organische Düngung und die Fruchtfolge gelegt. Kleine Zentren der Region stellten die Kirchdörfer dar. In diesen befanden sich die Schulen und hier waren verstärkt die Handwerker angesiedelt.

Das größte Gut des Ortes bekrönte ein Uhrturm. Vor den Pferdeställen wetteiferten die Bauern um die schönste Kumthalle. Der Stolz der Bäuerinnen stellte der Bauerngarten dar. Buchsbaumhecken gliederten die Beete und oft bildete eine Holzlattenlaube den Mittelpunkt dieses Refugiums.

Die Pflege von Hecken und Höhenbepflanzungen beugte der Erosion vor. Die Schlaggrößen und die Feldwege bewegten ein Übriges. Die selbstverständlichste Winterarbeit war, abgeschwemmten und am Feldrand abgelagerten Boden wieder auf das Feld zu fahren. Ein guter Bau- er wirtschaftete nach dem Grundsatz: Ich habe mein Land nicht von den Vätern ererbt, sondern von den Enkeln geliehen.

Die erste große Zäsur ergab sich nach dem Zweiten Weltkrieg. Eine Generation an Bauern war im Krieg geblieben. Es fehlten die Nachfolger. Dazu kam die Politik in der DDR, die gegen die Einzelbauern gerichtet war. Die Bodenreform, die LPG-Bildung und die veränderte Einstellung der Landeigentümer („Unsere Kinder gehen nicht in die LPG, sondern in die Industrie“) führten zu einer Entfremdung von Bewirtschafter und Eigentümer. Dies führte, auch durch die Intensivierung der Landwirtschaft, zum Freisetzen von Arbeitskräften und damit zum Wegzug bzw. Leerstand in den Dörfern. Ein weiteres Problem ergab sich durch die Konzentration der Produktionseinheiten. Die Güter erwiesen sich für die neue Technik und die Stallgrößen als zu klein. In einigen entstanden Wohnungen, andere verfielen und wurden in der Folge abgerissen.

In den LPGen entstand eine industriemäßige Produktion. In wenigen Dörfern, meist den Standorten der LPG, wurden neue Hallen für größere Ställe, Werkstatt- und Vermarktungskomplexe und natürlich Büro- und Sozialgebäude gebaut. Dazu entstanden in einigen Dörfern vorstadtähnliche Neubausiedlungen für die LPGMitglieder. Die junge Generation, die die Fachwerkhäuser der Eltern übernahm, baute natürlich auch um. Moderne Häuser mit großen, ausdrucklosen Fenstern entstanden. Die alten Bauerngärten und Streuobstwiesen benötigte keiner mehr. Von staatlicher Seite erhielten die LPGs Prämien für zusätzlich geschaffene landwirtschaftliche Nutzflächen. Feldwege und Feldraine wurden planiert und, wenn es die Hangneigung zuließ, Hecken und Streuobstwiesen in Ackerflächen umgestaltet. Zusätzlich legte man ein umfangreiches Meliorationsprogramm für die Rekultivierung von Auenflächen auf. Dazu gründeten die LPGs in den Kreisen jeweils einen gemeinsamen Baubetrieb. Meliorationsoder Beregnungsprojekte legte man entsprechen der Plansituation und der anzubauenden Fruchtfolge fest. Die Landschaften wurden so zugunsten der Großraumwirtschaft „ausgeräumt“. Einsprüche von Landeigentümern gab 
es nicht, da sie LPG-Mitglieder waren oder Erbengemeinschaften angehörten.

In der Folge zeigten sich Erosionsrinnen von über einen Meter Tiefe. Gab es Probleme bei der Bearbeitung, schob eine Planierraupe die Rinnen zu und der Regen arbeitete neu. Für den Hochwasserschutz errichteten die Betriebe an exponierten Stellen zum Schutz der betreffenden Dörfer Dämme, deren Projektierung und Genehmigung problemlos erfolgte.

In der Folge diese Bewirtschaftung ging der Fischbestand in den Gewässern zurück. Feldhase, Fasan und Rebhuhn verschwanden. Das Jagdgesetz der DDR honorierte die Bekämpfung von Schadwild, wie Fuchs und Marder, aber auch von Krähen und Elstern. Dies führte zu einem guten Bestand an Rehwild. Der Schwarzwildbestand hielt sich in Grenzen. Der Bestand an Singvögeln war gut.

Die Aussagen können natürlich nicht pauschalisiert werden, da für das Bewirtschaftungsergebnis nicht unerheblich war, wie sich LPG-Vorsitzenden und der Vorstand zur Natur stellten.

Die Hoffnung, dass nach der Wende eine Verkleinerung der Schläge erfolgen würde, verflüchtigte sich schnell. Die Wiedereinrichter und Pächter nahmen die großen Schläge, die eine effektive Bewirtschaftung ermöglichten, gern an. In der Gemeinde mit 2.500 Hektar landwirtschaftlicher Fläche entstanden ein Feldgemüseproduzent, zwei Obstbauern, ein Schäfer und ein Rinderzüchter. Letzterer, ein Nebenerwerbslandwirt, beweidet mit seinen drei Muttertierherden die Auenflächen und, wie der Schäfer, jene Flächen, die nicht ackerbaulich genutzt werden können. Die übrigen Flächen nutzt man ausschließlich für den Feldbau. Dabei dominiert der Anbau von Getreide, Mais und Raps. Durch intensive Düngung erzielt man, trotz Monokultur, Spitzenerträge. Erosionsmindernde Maßnahmen oder Maßnahmen zur Bodenverbesserung bzw. zum Erhalt der Bodenwertzahl sind nicht in den Pachtverträgen verankert und deutschlandweit verteilte Erbengemeinschaften legen auch nur Wert auf einen maximalen Pachtpreis.

Von den über 200 Arbeitsplätzen in der LPG Feldbau und ähnlich vielen in der Tierzucht sowie 60 in der Gärtnerischen Produktionsgenossenschaft sind nur wenige übrig geblieben. Ohne Tierzucht rechnet man bei der Feldbestellung eine Arbeitskraft auf 100 bis 150 Hektar. Der Wegfall der Arbeitsplätze im ländlichen Raum hat dramatische Folgen. Die Jugend zieht weg. Es entsteht eine Überalterung.

Ein Familienbetrieb im Ländlichen Raum rechnet sich heute erst bei 400 Hektar! Der durchschnittliche Betrieb alter Prägung lag bei 30 Hektar. Das bedeutet, einem Wiedereinrichter oder wieder in Betrieb genommenen Vierseithof stehen 15 Abrisshöfe gegenüber. Da die Betriebsgrößen teils wesentlich größer sind, sind die Auswirkungen noch drastischer.

Die Altgemeinde Leuben-Schleinitz (seit 2015 Teil der Stadt Nossen) hat in den letzten 25 Jahren 40 Prozent der Einwohner eingebüßt. Dazu kommen Schließung der Schule, der Post und von Versorgungseinrichtungen. Die Gemeinde versuchte, dieser Tendenz entgegenzuwirken: Maßnahmen waren Flurneuordnung, Einrichtung von Förderdörfern, Sportplatzneubau, Sanierung der Turnhalle und der Kita sowie Abwasserbau und Neubau der Trinkwasserversorgung. Wir legten weiterhin größten Wert auf den Erhalt des Vereinslebens. Doch gerade hier schlägt die Entwicklung voll negativ zu. Jugendliche die nicht mehr da sind, können in keinen Verein gehen, und Kinder gehen in Vereine an ihrem auswärts gelegenen Schulstandort.

Die Umnutzung der Vierseithöfe ist keine flächendeckende Lösung, da sie lediglich Leuchttürme darstellen. Zusätzlich negativ wirkt sich das „Plattmachen“ des Ländlichen Raums durch die Behörden aus. Alle Dörfer wurden als Außenbereich eingestuft. Dadurch wird jegliche vorgesehene Bautätigkeit massiv behindert. Das betrifft Familien, die um- und ausbauen oder umnutzen wollen. Aber wie wollen wir Leben in den Dörfern erhalten, wenn alle Bautätigkeit massiv behindert oder verhindert wird?

Neben den Erosionsproblemen tickt neben der fehlenden Gewässerpflege und dem vollkommenden Ignorieren der zunehmenden Probleme aus der Vernachlässigung der Meliorationsarbeiten eine weitere Zeitbombe: der Umgang mit abfließendem Wasser und der Hochwasserschutz. Gewässer- und Bodenverbände, die Abhilfe schaffen könnten, sind in Sachsen nicht gewollt. Das Sächsische Wassergesetz behindert Lösungen. Da die Kommune die Dammpflege tätigen und gleichzeitig die zu überstauende Fläche an die Kommune übergehen soll, sind sowohl Kommune als auch Bauern nicht an solchen Maßnahmen interessiert. Bei dem Bau von Ablaufhindernissen und zeitweilig zu überstauenden Flächen, wie es uns unsere Altvorderen vormachten (Durchlässe, die Rückstau erzeugen usw.), sind unsere Behörden überfordert.

Weitere Probleme können sich ergeben, wenn, durch das Versagen der Förderpolitik, Schäfer und Rinderzüchter aufgeben. Wer pflegt dann die Auenflächen? Hier sind wir bei einem weiteren Problem: Bauern, Jäger, Umweltschützer und Behörden müssen an einem Strang ziehen. Man sollte sich aber vorher über die Richtung einigen. Die Natur kann ohne uns, wir aber nicht ohne die Natur.

\section{Autor}

Gerhard Doleschal 1991 bis 1993 Bürgermeister der Gemeinde Schleinitz, 1993 bis 2013 Bürgermeister der Gemeinde Leuben-Schleinitz 